\title{
AN OPTIMAL TIME CONTROL PROBLEM FOR THE ONE-DIMENSIONAL, LINEAR HEAT EQUATION, IN THE PRESENCE OF A SCALING PARAMETER
}

\author{
Karim Benalia $^{1}$, Claire David ${ }^{2}$ and Brahim Oukacha ${ }^{3}$
}

\begin{abstract}
In this paper, we study the optimal time problem for the one-dimensional, linear heat equation, in the presence of a scaling parameter. To begin with, we build an exact solution. The dependence of this solution as regards the scaling parameter naturally opens the way to study the existence and uniqueness of an optimal time control. If, moreover, one assumes the $L^{\infty}$ - null controllability, it enables to establish a bang-bang type property.
\end{abstract}

Mathematics Subject Classification. 35K05.

Received June 25, 2016. Accepted January 6, 2017.

\section{INTRODUCTION}

In an optimal time control problem, one searches the minimum time for an eligible control, in order to bring a dynamic system, from an arbitrary initial point, to a final prescribed one. This problem is classical in finite dimension, with nice properties, due to the maximum principle of Pontriaguine, and the bang-bang property (see the seminal article by Bellman, Glicksberg and Gross [1]).

The generalization in infinite dimension was initiated by Fattorini [2-4], and developed by Lions [5]. The book of Tucsnak and Weiss [6] is an unavoidable reference for the control.

The general optimal control problem for the equation of the linear heat-dimensional, on a compact $K \subset \mathbb{R}$, can be stated as follows:

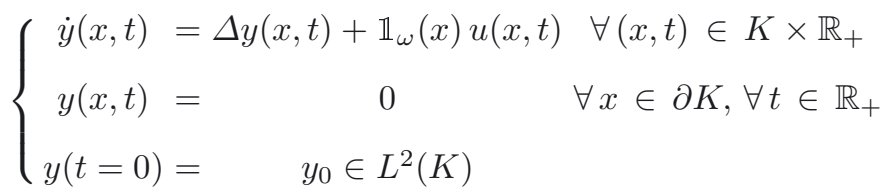

Keywords. Optimal time control problem, null controllability, bang-bang property, heat equation, Scaling parameter.

1 Laboratory of operational research and mathematical decision, Mouloud Mammeri University, Tizi-Ouzou, Algeria. benalia.karim@yahoo.fr

2 Laboratoire Jacques-Louis Lions,4, place Jussieu 75005, Paris, France, Sorbonne University, UPMC Univ Paris 06 CNRS, UMR 7598, Paris France. Claire.David@upmc.fr

3 Laboratory of operational research and mathematical decision, Mouloud Mammeri University, Tizi-Ouzou, Algeria.

oukachabrahim@yahoo.fr 
where $t \in[0, T]$ denotes time, $\omega \subset K$, while $\mathbb{1}_{\omega}$ is the characteristic function of the set $\omega$ :

$$
\forall x \in K: \quad \mathbb{1}_{\omega}(x)= \begin{cases}1 & \text { if } x \in \omega \\ 0 & \text { if not }\end{cases}
$$

where $u$ is in $L^{\infty}(\omega,[0, T])$, and where:

$$
\|u\|_{L^{\infty}(\omega,[0, T])} \leq 1 \quad \text { for almost all } t \in[0, T] .
$$

The objective for the problem (1.1) is to find the control $u^{\star}$ which is accessible, with a final time $\tau^{\star}$ as small as possible (see [2-4] and the references therein).

One can obtain the bang-bang property by means of the maximum principle, if the system can be controled exactly (see for example, the article of Lohéac and Tucsnak [7]).

It is now well known that for specific equations (for the general heat equation, for instance), the exact controllability is not verified. This is the reason why we choose to study new conditions, without using the maximum principle. With an assumption on the $L^{\infty}$ - null controllability, we can establish the bang-bang property (see also the paper of Micu, Roventa and Tucsnak [8], and the one of Wang [18]).

The objective of our work is to study the existence and uniqueness of an optimal time control problem for the one-dimensional, linear heat equation, in the presence of a scaling parameter, and to determine whether the bang-bang property can be satisfied.

The rest of the paper is organized as follows. In Section 2 we build the invariant solutions for the linear heat equation and prove the existence and uniqueness of the time optimal control for the heat equation, in the presence of a scaling parameter. In Section 3 we present a numerical results. Perspectives are presented in Section 4.

\section{Optimal time Control With a SCAling invariant SOlutions FOR THE ONE-DIMENSIONAL, LINEAR HEAT EQUATION}

We are interested in the following, to the linear, one-dimensional, normalized, heat equation:

$$
\frac{\partial y}{\partial t}=\frac{\partial^{2} y}{\partial x^{2}} \quad \forall(x, t) \in \mathbb{R} \times[0, T]
$$

where $T$ is a positive real number, with the initial condition:

$$
y(x, 0)=y_{0}(x) \quad \forall x \in \mathbb{R}
$$

and where $y_{0}$ denotes a given function.

The analytical solution is given, for any $(x, t) \in \mathbb{R} \times[0, T]$, by:

$$
y_{\text {classical }}(x, t)=\frac{1}{2 \sqrt{\pi t}} \int_{-\infty}^{\infty} y_{0}(u) \mathrm{e}^{-\frac{(x-u)^{2}}{4 t}} \mathrm{~d} u .
$$

The linear heat equation has a natural scaling invariance. Let us denote by $y$ a solution. Then, for any strictly positive real number $\Lambda$, the mapping:

$$
(t, x) \mapsto y_{\Lambda}(t, x)=\Lambda y\left(\Lambda^{2} t, \Lambda x\right)
$$

is also a solution. 
By applying the method developed by Jean-Yves Chemin and Claire David [10,11], one can introduce the mapping $\mathcal{F}$, belonging to $L_{\text {loc }}^{2}(\mathbb{R}) \times \mathbb{R}_{+}^{\star} \times \mathbb{N}^{\star}$, by:

$$
\mathcal{F}\left(y_{0}, \Lambda, N_{0}\right)=y_{0}+\varepsilon \sum_{j=1}^{N_{0}} \Lambda^{-j} y_{0}\left(\Lambda^{-j} \cdot\right), \quad \varepsilon \in\{-1,+1\}, N_{0} \in \mathbb{N}^{\star} .
$$

The building of thus mapping takes its origin in the profile theory, introduced by Gérard et Bahouri [12]. It is based on the idea that two solutions of an evolution equation, of scales, sufficiently different, almost not interact. We are thus interested, in the following, to initial data of the form:

$$
y_{0}(x)+\varepsilon \sum_{j=1}^{N_{0}} y_{0, \Lambda, j}(x)=y_{0}(x)+\varepsilon \sum_{j=1}^{N_{0}} \frac{1}{\Lambda^{j}} y_{0}\left(\frac{x}{\Lambda^{j}}\right), \quad \Lambda>0 .
$$

The exact analytical solution $\tilde{y}$, which depends on the space variable $x$, the time variable $t$, and the scaling parameter $\Lambda$, is given by:

$$
\tilde{y}(x, t, \Lambda)=y_{\text {classical }}(x, t)+\varepsilon \frac{1}{2 \sqrt{\pi t}} \int_{-\infty}^{\infty} \sum_{j=1}^{N_{0}} y_{0, \Lambda, j}(u) \mathrm{e}^{-\frac{(x-u)^{2}}{4 t}} \mathrm{~d} u .
$$

It is interesting to note that:

$$
\tilde{y}(x, t, \Lambda)=y_{\text {classical }}(x, t)+\varepsilon \sum_{j=1}^{N_{0}} \frac{1}{\Lambda} y_{\text {classical }}\left(\frac{x}{\Lambda^{j}}, \frac{t}{\Lambda^{2 j}}\right) .
$$

Hence, we have:

$$
\Delta \tilde{y}(x, t, \Lambda)=\Delta y_{\text {classical }}(x, t)+\frac{\varepsilon}{\Lambda} \sum_{j=1}^{N_{0}} \Delta y_{\text {classical }}\left(\frac{x}{\Lambda^{j}}, \frac{t}{\Lambda^{2 j}}\right) .
$$

One builds thus an exact solution of the afore mentioned linear heat equation. The dependence of this solution towards the scaling parameter $\Lambda$, naturally leads to an internal control problem, which can be formulated as follows: being given a compact $K \subset \mathbb{R}$, and a domain $\omega \subset K$, what kind of control $u$ does one require in order to obtain a given result? The associated system is the following:

$$
\left\{\begin{array}{lrl}
\dot{\tilde{y}}(x, t, \Lambda) & =\Delta \tilde{y}(x, t, \Lambda)+\mathbb{1}_{\omega}(x) u(x, t) & \forall(x, t, \Lambda) \in K \times \mathbb{R}_{+} \times \mathbb{R}_{+}^{\star} \\
\tilde{y}(x, t, \Lambda)=0 & \forall x \in \partial K, \forall(t, \Lambda) \in \mathbb{R}_{+} \times \mathbb{R}_{+}^{\star} \\
\tilde{y}(x, 0, \Lambda)=\tilde{y}_{0} \in L^{2}(K) & \forall \Lambda \in \mathbb{R}_{+}^{\star}
\end{array}\right.
$$

where $\mathbb{1}_{\omega}$ is the characteristic function of $\omega$ :

$$
\forall x \in K: \quad \mathbb{1}_{\omega}(x)= \begin{cases}1 & \text { if } \quad x \in \omega \\ 0 & \text { if not }\end{cases}
$$

and where $u$ is a control checking:

$$
\forall t \geq 0: \quad\|u\|_{L^{2}(K)} \leq C_{0}
$$

while $C_{0}$ denotes a positive constant, that depends on the initial condition $\tilde{y}_{0}$. In the following, this initial condition will be adjusted in order to have:

$$
C_{0} \leq 1 .
$$


Let us recall the following classical results:

Proposition 2.1. For any strictly positive real time $T$, and for any continuous function $u$ defined on $\omega \times[0, T]$, the unique (mild) solution y such that:

$$
y(t=0, \cdot)=y_{0}(\cdot)
$$

is given, thanks to the Duhamel formula, for any couple $(x, t)$ belonging to $K \times[0, T]$, by:

$$
y(x, t)=\mathrm{e}^{t \Delta} y_{0}(x)+\int_{0}^{t} \mathrm{e}^{(t-\tau) \Delta} \mathbb{1}_{\omega}(x) u(\tau, x) \mathrm{d} \tau .
$$

Proposition 2.2. For any strictly positive number $T$, and for any continuous function $u$ of $L^{2}(\omega \times[0, T])$, the system (2) has a unique solution $\tilde{y}$ such that:

$$
\tilde{y} \in C\left([0, T], H_{1}^{0}(K)\right), \quad \frac{\partial \tilde{y}}{\partial t} \in L^{2}\left([0, T], L^{2}(K)\right) .
$$

As recalled in [13], it is then natural to introduce, for any strictly positive number $T$, the set of reachable states at time $T$ related to the system (2):

$$
\mathcal{R}(T)=\left\{\tilde{y}(T) \mid u \in L^{2}(K \times[0, T])\right\} .
$$

Proposition 2.3 [13]. For any strictly positive number $T$, the set $\mathcal{R}(T)$ is dense in $L^{2}(K)$.

Proof. The set $\mathcal{R}(T)$ is obviously a vectorial subspace of $L^{2}(K)$.

Let us concentrate on the orthogonal of the set $\mathcal{R}(T)$ in $L^{2}(K)$. To this purpose, let us consider the retrograde problem:

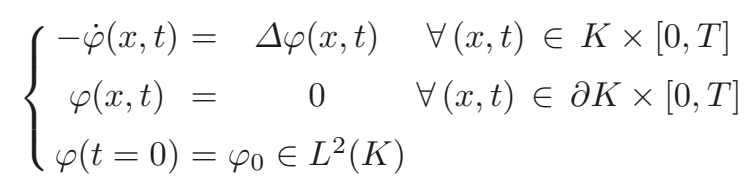

This problem, which brings back to the equation of the classical heat through the of variable

$$
t \mapsto T-t
$$

admits a unique solution $\varphi$ in

$$
C\left([0, T], L^{2}(K)\right) \cap L^{2}\left([0, T], H_{1}^{0}(K)\right)
$$

with:

$$
\frac{\partial \varphi}{\partial t} \in L^{2}\left([0, T], H^{-1}(K)\right) .
$$

By multiplying by $\varphi$ each member of the relation:

$$
\dot{y}=\Delta y+\mathbb{1}_{\omega} u
$$

one gets:

$$
\varphi \dot{y}=\varphi \Delta y+\varphi \mathbb{1}_{\omega} u
$$


Integration leads to:

$$
\int_{0}^{T} \int_{K} \varphi \dot{y} \mathrm{~d} x \mathrm{~d} t=\int_{0}^{T} \int_{\partial K} \varphi \Delta y \mathrm{~d} x \mathrm{~d} t \int_{0}^{T} \int_{K} \varphi u \mathrm{~d} x \mathrm{~d} t
$$

i.e.:

$$
\int_{0}^{T} \int_{K} \varphi \dot{y} \mathrm{~d} x \mathrm{~d} t=-\int_{0}^{T} \int_{K} \frac{\partial \varphi}{\partial x} \frac{\partial y}{\partial x} \mathrm{~d} x \mathrm{~d} t+\int_{0}^{T} \int_{K} \varphi u \mathrm{~d} x \mathrm{~d} t
$$

or:

$$
\int_{0}^{T} \int_{K} \varphi \dot{y} \mathrm{~d} x \mathrm{~d} t=-\int_{0}^{T} \int_{\partial K} \frac{\partial \varphi}{\partial x} y \mathrm{~d} x \mathrm{~d} t+\int_{0}^{T} \int_{K} \frac{\partial^{2} \varphi}{\partial x^{2}} y \mathrm{~d} x \mathrm{~d} t+\int_{0}^{T} \int_{K} \varphi u \mathrm{~d} x \mathrm{~d} t
$$

i.e.:

$$
\int_{0}^{T} \int_{K} \varphi \dot{y} \mathrm{~d} x \mathrm{~d} t=\int_{0}^{T} \int_{K} \frac{\partial^{2} \varphi}{\partial x^{2}} y \mathrm{~d} x \mathrm{~d} t+\int_{0}^{T} \int_{K} \varphi u \mathrm{~d} x \mathrm{~d} t
$$

since $\varphi$ and $y$ take zero values on the boundary $\partial K$. An integration by part leads then to:

$$
\int_{K} y(x, T) \varphi(x, T) \mathrm{d} x-\int_{0}^{T} \int_{K} \dot{\varphi} y \mathrm{~d} x \mathrm{~d} t=\int_{0}^{T} \int_{K} \frac{\partial^{2} \varphi}{\partial x^{2}} y \mathrm{~d} x \mathrm{~d} t+\int_{0}^{T} \int_{\omega} \varphi u \mathrm{~d} x \mathrm{~d} t .
$$

By taking into account:

$$
-\dot{\varphi}=\Delta \varphi
$$

one gets:

$$
\int_{K} y(x, T) \varphi(x, T) \mathrm{d} x=\int_{0}^{T} \int_{\omega} \varphi u \mathrm{~d} x \mathrm{~d} t
$$

As

$$
\varphi(\cdot, T)=\varphi_{0} \in \mathcal{R}(T)^{\perp}
$$

one deduces:

$$
\int_{0}^{T} \int_{\omega} \varphi u \mathrm{~d} x \mathrm{~d} t=\int_{K} y(x, T) \varphi(x, T) \mathrm{d} x=0 .
$$

Since one can take any control function $u$, we have:

$$
\varphi=0 \quad \text { in } \quad K \times[0, T] .
$$

The generalized result of Mizohata [14] allows then to deduce:

$$
\varphi=0 \quad \text { in } \quad K \times[0, T]
$$

Thus:

$$
\varphi_{0}=0
$$

The orthogonal of $\mathcal{R}(T)$ in $L^{2}(K)$ is then reduced to $\{0\}$.

One can then apply the density criterion, the one which is a corollary of the Hahn-Banach theorem, which states that if $E$ is a normed vector space, and $F$ a subspace of $E$, such that any continuous linear form on $E$ vanishes on $F$, then, $F$ is dense in $E$. 
Thus, since:

$$
\left(\mathcal{R}(T)^{\perp}\right)_{\perp}=\left\{\varphi \in L^{2}(K) \mid \forall \psi \in \mathcal{R}(T)^{\perp}: \quad\langle\varphi, \psi\rangle=0\right\}=\overline{\mathcal{R}(T)}
$$

this allows to state that the necessary and sufficient condition of density of $\mathcal{R}(T)$ in $L^{2}(K)$ :

$$
\overline{\mathcal{R}(T)}=L^{2}(K)
$$

is therefore equivalent to:

$$
\mathcal{R}(T)^{\perp}=\{0\} .
$$

Corollary 2.4. Let us consider $\tilde{y}$ in $L^{2}(K)$, and a strictly positive number $\varepsilon$. There exists a control u, belonging to $L^{2}(K \times[0, T])$, such that:

$$
\|y(\cdot, T)-\tilde{y}\|_{L^{2}(K)} \leq \varepsilon .
$$

Remark 2.5. Thus, the study of the approximate controllability of our linear system (2.1) reduces to a continuity problem, for the associate adjunct problem.

We now aim at determining if we there is a control $u$ which verifies the following bang-bang property:

$$
\|u(., t)\|_{L^{2}(K)}=1 \quad \text { for all } t \in\left[0, \tau^{\star}\right]
$$

where $\tau^{\star}$ is a unique optimal time that can lead the solution of (2.1) from the starting point $\tilde{y}_{0}$ to $\tilde{y}^{f}$, at the time $\tau^{\star}$. This enables one to go back to the work of Lebeau et Robbiano [15], which leads to the following result:

Proposition 2.6. For any $\tilde{y}_{0}$, and for any $\tilde{y}^{f}$ in $L^{2}(K)$ such that

$$
\tilde{y}_{0} \neq \tilde{y}^{f}
$$

if $\tilde{y}$ is a solution of system (2.1), associated with $u^{\star}$, there is a unique optimal time $\tau^{\star}$ which leads the solution of (2.1), from the starting point $\tilde{y}_{0}$, to $\tilde{y}^{f}$, at the time $\tau^{\star}$.

Moreover, $u^{\star}$ checks the bang-bang property, i.e.:

$$
\|u(., t)\|_{L^{2}(K)}=1 \quad \text { for all } t \in\left[0, \tau^{\star}\right]
$$

Proof. The proof of the proposition can be found in [18].

\section{Numerical Results}

Our numerical application is carried out by means of a direct type method (total discretization), of the afore mentioned linear heat equation, in the presence of a scaling parameter $\Lambda>0$, with an internal control, in a domain $\omega \subset[0,1]$.

More precisely, we consider the following system:

$$
\begin{gathered}
\Lambda^{2} \dot{y}\left(\Lambda x, \Lambda^{2} t\right)=\Lambda^{2} \Delta y\left(\Lambda x, \Lambda^{2} t\right)+\mathbb{1}_{\left[\frac{1}{3}, \frac{2}{3}\right]}(x) u(x) \quad \forall x \in[0,1], \forall t \in\left[0, t_{f}\right], \\
y(0, t, \Lambda)=0, \quad y(1, t, \Lambda)=0, \quad \forall t \in\left[0, t_{f}\right], \Lambda>0 .
\end{gathered}
$$

The discretization is carried out by finite differences, with an implicit Euler scheme in time.

To this purpose, let us consider the time discretization:

$$
0=t_{0}<t_{1}<\ldots<t_{i}<\ldots<t_{n}=t_{f}
$$


and the space discretization:

$$
0=x_{0}<x_{1}<\ldots<x_{j}<\ldots<x_{N+1}=1 .
$$

For any integer $i$ belonging to $\{0, \ldots, n\}$, and for any $j$ belonging to $\{0, \ldots, N+1\}$, let us denote by:

$$
y_{i, j}=y_{\Lambda}\left(t_{i}, x_{j}\right)
$$

the value of the solution at $t=t_{i}$ and $x=x_{j}$, for the scaling parameter $\Lambda$.

We assume:

$$
\dot{y}_{\lambda}\left(t_{i}, x_{j}\right) \approx \frac{y_{i, j}-y_{i-1, j}}{t_{h}}
$$

and:

$$
\Delta y_{\Lambda}\left(t_{i}, x_{j}\right) \approx \frac{y_{i, j+1}+y_{i, j-1}-2 y_{i, j}}{x_{h}^{2}}
$$

where:

$$
t_{h}=\frac{t_{f}}{n}, \quad x_{h}=\frac{\Lambda}{N+1}, \quad t_{f}=\Lambda^{2} .
$$

For each integer $i$ belonging to $\{1, \ldots, N\}$, we set:

$$
Y_{i}=\left(\begin{array}{c}
y_{i, 1} \\
y_{i, 2} \\
\vdots \\
y_{i, N}
\end{array}\right) .
$$

The related discrete system can be written under the following matrix form:

$$
\Lambda^{2} \frac{Y_{i+1}-Y_{i}}{t_{h}}=A_{h} \cdot Y_{i+1}+B_{h} \cdot U_{i+1}
$$

where the $N \times N$ matrix $A_{h}$ is given by:

$$
A_{h}=\frac{\Lambda^{2}}{x_{h}^{2}}\left(\begin{array}{ccccc}
-2 & 1 & 0 & \ldots & 0 \\
1 & -2 & \ddots & & \vdots \\
0 & \ddots & \ddots & \ddots & 0 \\
\vdots & & \ddots & -2 & 1 \\
0 & \ldots & 0 & 1 & -2
\end{array}\right)
$$

and where the $N \times N$ diagonal matrix $B_{h}$ is given by:

$$
B_{h}=\operatorname{diag}\left(\alpha_{1}, \ldots, \alpha_{N}\right)
$$

while, for any integer $i$ belonging to $\{1, \ldots, N\}$ :

$$
\alpha_{i}=\left\{\begin{array}{l}
1 \quad \text { if } \quad \frac{1}{3} \leq x_{i} \leq \frac{2}{3} \\
0 \text { otherwise }
\end{array}\right.
$$


and:

$$
U_{i+1}=\left(\begin{array}{c}
u_{i, 1} \\
\vdots \\
u_{i, N}
\end{array}\right)
$$

where $u_{i, j}$ is the value chosen for the control at $t=t_{i}$ and $x=x_{j}$.

Basic calculations enable one to solve, for all $i$ in $\{1, \ldots, N\}$, the system equivalent to (3.3):

$$
C Y_{i+1}=Y_{i}+\frac{t_{h}}{\Lambda^{2}} B_{h} \cdot U_{i}
$$

where

$$
C=I_{N}-\frac{1}{\Lambda^{2}} t_{h} \cdot A_{h}
$$

$I_{N}$ denoting the $N \times N$ identity matrix.

At each time step, the matrix $C$ is inverted, in order to calculate $Y_{i+1}$.

Let us denote by $X$ a variable which contains the whole set of values

$$
\left\{u_{i, j} \mid i \in[1, N], j \in[1, N]\right\} \cup\left\{t_{f}\right\} .
$$

One has to bear in mind that the principle of direct methods lay in minimizing a function $F$ that yields $t_{f}$ with constraints, i.e., for each integer $i$ of $\{1, \ldots, N\}$ :

$$
\left\|U_{i}\right\|_{L^{2}\left(\left[\frac{1}{3}, \frac{2}{3}\right]\right)} \leq 1
$$

with the final condition:

$$
Y\left(t_{f}\right)=Y^{f}
$$

The initial and final conditions are:

$$
\forall x \in K: \quad y_{0}(x)=\sin (\pi x), \quad y^{f}(x)=0 .
$$

Our simulation is carried for:

$$
n=N \text {. }
$$

In practice, we choose the number of discretizations ( $N=10$ for instance), while changing the value of the scaling parameter. Numerical results are given in Table 1.

\section{Comparison with a shooting method (The indirect method).}

To transform the problem (3.1)-(3.2) into a control problem governed by ordinary differential equations, we discretize the heat equation (3.1) in the spatial direction $x$. By dividing the interval $[0,1]$ into $N$ intervals of length $x_{h}=\frac{\Lambda}{N}$, we obtain the following optimal control problem, governed by ordinary differential equations:

$$
\begin{gathered}
\min \int_{0}^{t_{f}} \mathrm{~d} t \\
\dot{Y}_{i}(t)=A_{h} Y_{i}(t)+B_{h} U_{i}(t), \quad Y_{i}(0)=Y_{i_{0}}, \forall t \in\left[0, t_{f}\right]
\end{gathered}
$$

where $A_{h}$ and $B_{h}$ are defined in (3.3).

The shooting method technique [19] for the above system leads to the adjoint system solution, and the solution $Y_{i}$ with the final condition $Y_{i}\left(t_{f}\right)=0$ 
TABle 1. Final time - Optimal time.

\begin{tabular}{l|cccccc}
\hline$\Lambda$ & 1 & 2 & 8 & 10 & 15 & 20 \\
\hline$\tau^{\star}$ (Total discretization method) & 0.6129 & 2.5506 & 44.8033 & 63.9311 & 79.1337 & 109.8435 \\
$\tau^{\star}$ (Shooting method) & 0.6013 & 2.5089 & 43.9041 & 62.7097 & 77.8107 & 109.1337 \\
\hline
\end{tabular}
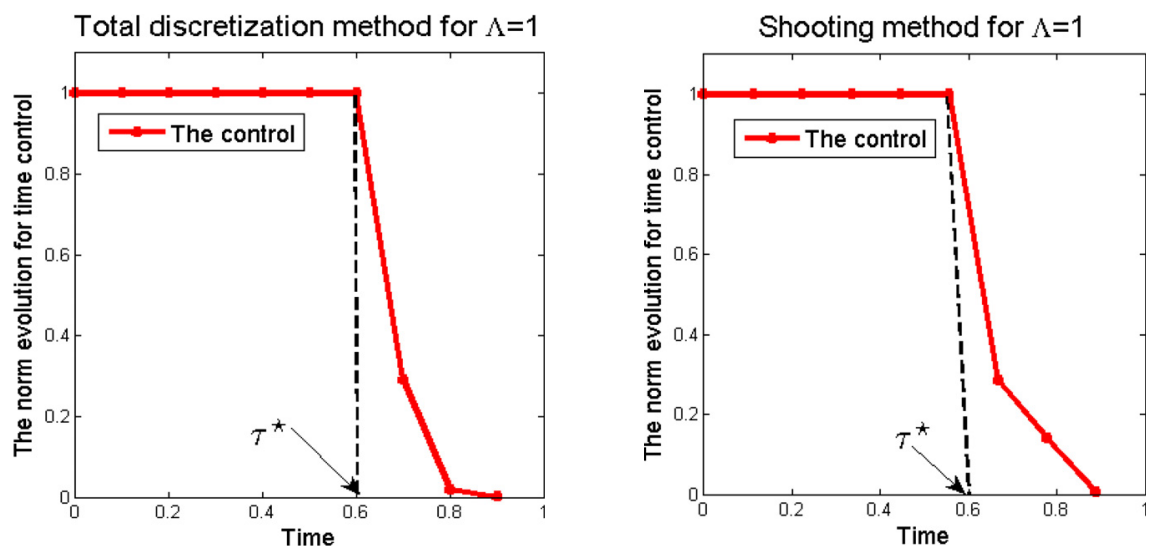

FIgURE 1. The norm evolution $L^{2}$ for time control with $\Lambda=1$.
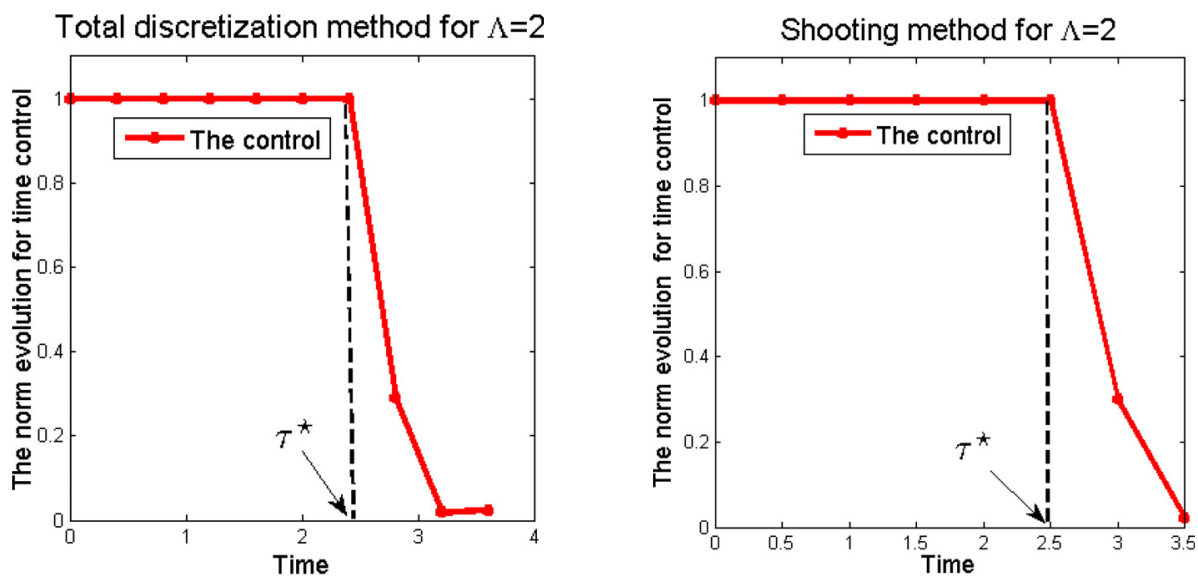

FIGURE 2. The norm evolution $L^{2}$ for time control with $\Lambda=2$.

Our results are given in Table 1. Comparison with the shooting method shows that the numerical results are very close to each other.

The following figures show the evolution of the $L^{2}$ norm of the time control using both methods, for different values of the scaling parameter. As said before, the results appear to be very close.

It is interesting to note that the $L^{2}$ norm of the time control take the value 1 for any time $t$ in $\left[0, \tau^{\star}\right]$. Thus, the control has the bang-bang property. Moreover, as the scaling parameter $\Lambda$ increases, so does the numerical final time, while the bang-bang property is still verified. 

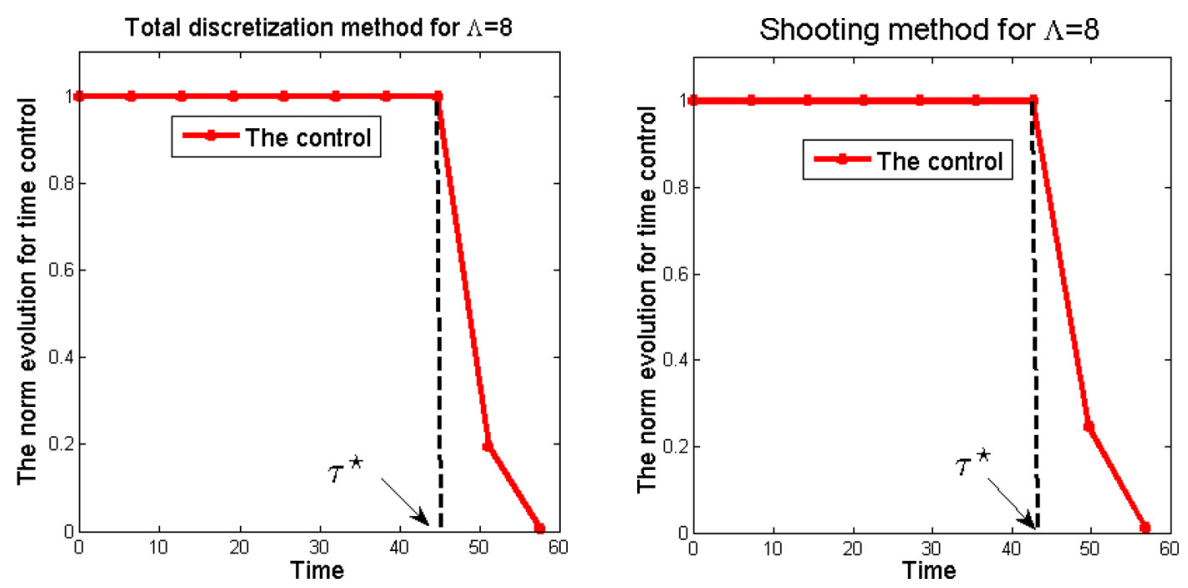

Figure 3 . The norm evolution $L^{2}$ for time control with $\Lambda=8$.
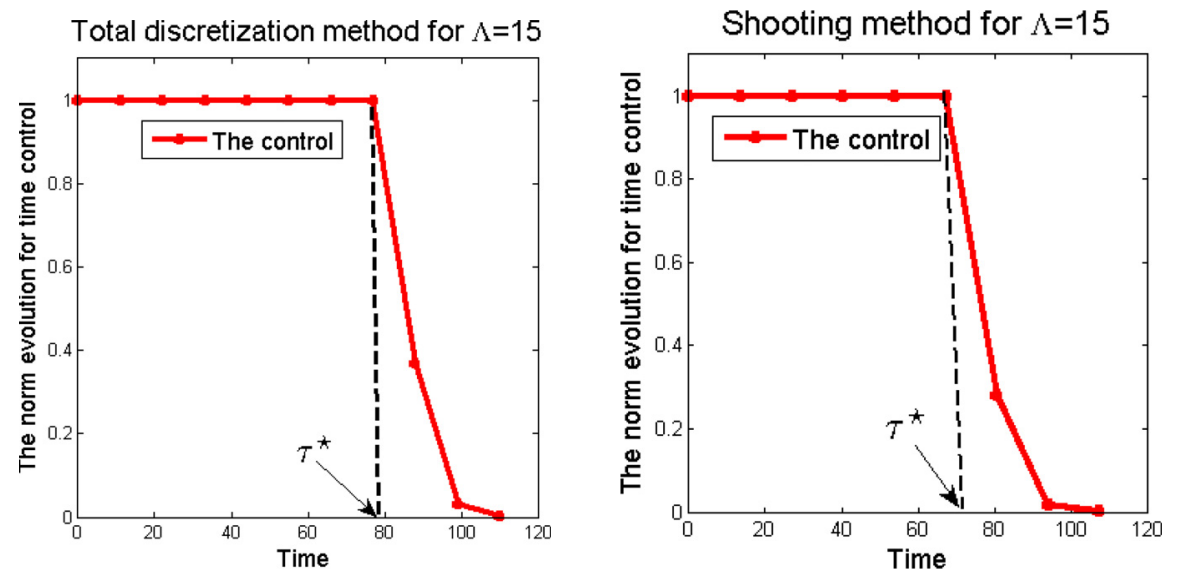

Figure 4. The norm evolution $L^{2}$ for time control with $\Lambda=15$.

\section{Perspectives}

Our approach has conventionaly, consisted in studying a system with an internal control, in the presence of a scaling parameter. It is interesting to note that, due to the expression of the solution with parameter:

$$
\tilde{y}(x, t, \Lambda)=y_{\text {classical }}(x, t)+\varepsilon \sum_{j=1}^{N_{0}} \frac{1}{\Lambda^{j}} y_{\text {classical }}\left(\frac{x}{\Lambda^{j}}, \frac{t}{\Lambda^{2 j}}\right)
$$

it appears interesting to consider a control of the form:

$$
\varepsilon \sum_{j=1}^{N_{0}} \frac{u_{j}}{\Lambda^{j}} y_{\text {classical }}\left(\frac{x}{\Lambda^{j}}, \frac{t}{\Lambda^{2 j}}\right)
$$


This thus leads to an affine control system. For any integer $j$ belonging to $\left\{1, \ldots, N_{0}\right\}$, the control $u_{j}$ corresponds to a displacement in the direction

$$
f_{j}=\varepsilon \frac{1}{\Lambda^{j}} y_{\text {classical }}\left(\frac{x}{\Lambda^{j}}, \frac{t}{\Lambda^{2 j}}\right)
$$

It is then natural to study the Lie algebra generated by the family

$$
\left(f_{j}\right)_{1 \leq i \leq N_{0}}
$$

in the spirit of what is presented in $[16,17]$, in so far the displacements on subintervals of $K$, in the given directions $f_{i}, f_{j}, i \neq j$, involve their Lie bracket.

\section{REFERENCES}

[1] R. Bellman, I. Glicksberg and O. Gross, On the "bang-bang" control problem. Quart. Appl. Math. 14 (1956) 11-18.

[2] H.O. Fattorini, Time-optimal control of solutions of operational differenital equations. J. Soc. Ind. Appl. Math. Ser. A Control 2 (1964) 54-59.

[3] H.O. Fattorini, A remark on the "bang-bang" principle for linear control systems in infinite dimensional spaces. SIAM J. Control 6 (1968) 109-113.

[4] H.O. Fattorini and H.O. Fattorini. Infinite dimensional linear control systems. The time optimal and norm optimal problems. Vol. 201 of North-Holland Mathematics Studies. Elsevier Science B.V., Amsterdam (2005).

[5] J.-L. Lions, Contrôle optimal de systèmes gouvernés par des équations aux dérivées partielles, Avant propos de P. Lelong. Dunod, Paris (1968).

[6] Tucsnak and Weiss, Observation and control for operator semigroups. Birkhäuser Advanced Texts: Basler Lehrbücher. [Birkhäuser Advanced Texts: Basel Textbooks]. Birkhäuser Verlag, Basel (2009).

[7] J. Lohéac and M. Tucsnak, Maximum principle and bang-bang property of time optimal controls for Schrödinger type systems. SIAM J. Control Optim. 51 (2013) 4016-4038.

[8] S. Micu, I. Roventa and M. Tucsnak, Time optimal boundary controls for the heat equation. J. Function. Anal. 263 (2012) $25-49$.

[18] G. Wang, $L^{\infty}$-null controllability for the heat equation and its consequences for the time optimal control problem. SIAM J. Control Optim. 47 (2008) 1701-1720.

[10] J.-Y. Chemin and Cl. David, Sur la construction de grandes solutions pour des équations de Schrödinger de type "masse critique", Séminaire Laurent Schwartz - EDP et applications (2013).

[11] J.-Y. Chemin and Cl. David, From an initial data to a global solution of the nonlinear Schrödinger equation: a building process. Int. Math. Res. Not. 2016 (2016) 2376-2396.

[12] H. Bahouri and P. Gérard, High frequency approximation of solutions to critical nonlinear wave equations. Amer. J. Math. 112 (1999) 131-175.

[13] J.-P. Puel, Contrôle et équations aux dérivées partielles. Journées mathématiques X-UPS (1999) 169-188.

[14] Unicité du prolongement des solutions pour quelques opérateurs paraboliques. Mem. Coll. Sci. Univ. Kyoto, Ser. A31 (1958) 219-239.

[15] G. Lebeau and L. Robbiano, Contrôle exact de l'équation de la chaleur. Commun. Partial Differ. Equ. 20 (1995) 335-356.

[16] J.-M. Coron, Quelques résultats sur la commandabilité et la stabilisation des systèmes non linéaires. Journées mathématiques X-UPS (1999) 123-168.

[17] Wei Liang Chow, Uber Systeme von Linearen Partiellen Differentialgleichungen Erster Ordnung. Math. Ann. 117 (1939) 98-105.

[18] L. Wang, Qishu Yan Bang-bang property of time optimal controls for some semilinear heat equation. J. Optim. Theory Appl. 165 (2015) 263278.

[19] E. Trélat. Contrôle optimal. Mathématiques Concrètes (Concrete Mathematics). Théorie 6$\}$ applications (Theory and applications). Vuibert, Paris (2005). 